\title{
Typical deterministic and stochastic bridge deterioration modelling incorporating backward prediction model
}

\author{
G. P. Bu ${ }^{1}$, J. B. Son ${ }^{1}$, J. H. Lee ${ }^{2}$, H. Guan ${ }^{1}$, M. Blumenstein ${ }^{3}$ and Y. C. Loo ${ }^{1}$ \\ ${ }^{1}$ Griffith School of Engineering, Gold Coast Campus, Griffith University, QLD \\ 4222, Australia \\ ${ }^{2}$ Centre for Infrastructure Engineering \& Management, Gold Coast Campus, \\ Griffith University, QLD 4222, Australia \\ ${ }^{3}$ School of Information and Communication Technology, Gold Coast Campus, \\ Griffith University, QLD 4222, Australia \\ *Tel: +61 (07) 55529284 \\ *Fax: +61 (07) 55528065 \\ *E-mail: g.bu@griffith.edu.au
}

\begin{abstract}
A Backward Prediction Model (BPM) has been developed to generate the missing bridge condition ratings in past years thereby ensuring adequate condition data as required in long-term performance modelling. The BPM establishes a correlation between the known condition ratings and the non-bridge factors including climate condition, traffic volume and population growth. The aim of this study is to confirm the ability of BPM in improving the prediction accuracy using the existing bridge deterioration models. The prediction accuracies of typical deterministic and stochastic bridge deterioration models are compared when different sets of BPM-generated historical condition ratings are used as input. Comparisons indicate that the prediction error decreases as more historical condition ratings are made available. Notwithstanding the above findings, several limitations of the current deterministic and stochastic bridge deterioration models are also worth noting and further research is essential to improve the prediction accuracy of bridge deterioration modelling.
\end{abstract}

Keywords Backward Prediction Model (BPM), deterioration modelling, deterministic models, stochastic models.

\section{Introduction}

In bridge management, critical decision-making for Maintenance, Repair and Rehabilitation (MR\&R) activities is required to ensure optimum levels of safety and serviceability of a bridge [1]. Many Bridge Management Systems (BMSs), as a Decision Support System (DSS), have been developed during the past decades 
for effective management of large bridge networks. A BMS, generally assisting significant future MR\&R strategies, is based on a reliable bridge deterioration model. The prediction accuracy of deterioration ratings is therefore highly crucial for an effective BMS [2]. A number of bridge deterioration models have been developed to determine the bridge life cycle for major MR\&R needs [3]. Nevertheless, the predictions of future structural condition ratings from BMSs are still impractical for developing long-term maintenance strategies. This is largely due to several drawbacks related to their applications in most bridge agencies, viz: (1) commercial BMS software has been implemented for only two decades, therefore bridge agencies would only have around 8 to 9 biennial inspection records [4]; (2) bridge condition ratings usually do not change significantly during short-term periods [4]; and (3) approximately $60 \%$ of BMS analytical modules rely heavily on periodical bridge inspection records [5]. These factors underlie the inaccuracy in predicting the future performance of bridges. In response, research efforts were made in the area of bridge asset management in an attempt to improve overall quality of BMS outcomes $[1,3]$.

Despite previous research achievements in the development of the deterioration models, some fundamental problems still remain. The most critical one is that the bridge inspection records are inadequate as required for BMS input. Many researchers and infrastructure asset management practitioners have recognised that deterioration of infrastructure facilities is not deterministic [6]. For effective implementation of BMS software, two important research problems must be solved: (1) As part of BMS data requirements, the amount of time-dependent bridge data from periodic bridge inspections for a BMS update is very limited; (2) Bridge condition rating variances in the existing small number of historical data cause inaccurate prediction results from the most important BMS analysis modules that require lengthy historical data patterns for their future projections. Typical deterioration modeling techniques have invariably been developed based on a few set of current structural condition ratings, thus unlikely to predict reliable future bridge condition ratings [4].

In addition to the above, bridge deteriorations are also caused by diverse explanatory variables including "non-bridge factors" such as traffic intensity, environmental factors, climates etc [7,8]. Collection of such variables is not included in the current bridge inspection routines. Because of this and with only 
limited bridge condition rating records, it is very difficult to use typical stochasticbased deterioration models to accurately predict future condition ratings. This limitation has been recognised and it is a challenging problem that has not yet been adequately addressed $[9,10,11,12]$.

As mentioned above, a deterioration model in a BMS is the most critical component and is based on historical condition ratings obtained from the elementlevel bridge inspections [5]. Although most bridge authorities have previously conducted inspection and maintenance tasks, these past inspection records are incompatible with what are required by a BMS as input. Such incompatibility is a major cause for the deficiency of the current BMS outcomes. Due to the lack of historical condition rating information, many bridge authorities worldwide have similar problems in using BMS for accurate and reliable predictions of long-term bridge performance and budget planning.

In order to address the problem of insufficient condition data, the Artificial Neural Network (ANNs) based-Backward Prediction Model (BPM) has been developed by Lee et al [4] for reliable bridge deterioration predictions. The BPM can generate missing condition ratings through a correlation between the existing condition ratings and the non-bridge factors which influence the variation of bridge condition ratings. This paper presents a comparison study using typical deterministic and stochastic deterioration modeling techniques incorporated with different numbers of BPM-generated condition rating data, in an attempt to improve the reliability of long-term prediction.

\section{Backward prediction model}

The BPM predicts the selected or entire periods of historical bridge condition ratings to overcome the problem of lacking BMS condition rating data. Figure 1 illustrates the main function of the ANN technique in establishing the correlation between the existing condition rating datasets (from year $m$ to $m+n$ ) and the corresponding years' non-bridge factors. The non-bridge factors directly and indirectly affect the variation of the bridge conditions and therefore the deterioration rate. The correlations established using neural networks are then applied to the non-bridge factors (for year 0 to $m$ ) to generate the missing bridge condition ratings (for the same year 0 to $m$ ). Thus, the non-bridge factors, in conjunction with the ANN technique, can produce the historical trends that help 
generate the missing condition ratings [4]. Detailed structure of the ANN-based BPM can be found in [4]. Note in this study that only six highly relevant nonbridge factors are employed including passenger vehicle, truck, total number of vehicles, maximum temperature, local city population and state population growth. All these non-bridge factors are relevant to the bridge network.

\section{BMS and NBI condition ratings for BPM}

\subsection{BMS condition ratings as BPM input}

The results obtained from the BPM were validated by using both backward and forward comparison techniques. The former compares the BPM outcomes with the known historical data to assess the prediction accuracy; whereas the latter uses the BPM outcomes as input data to predict present year's bridge condition ratings, which are then compared directly with the actual data in the corresponding year [13]. To carry out the backward comparison, only five sets of the existing BMS and National Bridge Inventory (NBI) condition ratings are used in this study as BPM training inputs and outputs (from 1996 to 2004 in a 2-year increment). Missing historical condition ratings can be generated from years 1968 to 1994 in a 2-year increment. Details of the BMS and NBI raw data as prediction inputs are given in Table 1 and Figure 2, respectively. The BMS raw data (from year 1996 to 2004 with a total quantity of 350) are those related to Element \#234 (i.e. reinforced concrete pier cap) on Bridge \#0301 xxxx1 and the NBI data (from year 1996 to 2004) are related to the bridge component (i.e. deck) of the same bridge. Note in Table 1 that the percentage values shown in brackets are the percentage quantities with respect to the total quantity. Note also that the Condition States (CSs) are used to quantify the severity and extent of damage for each bridge element. A five-CS scale is adopted in Queensland, Australia [14]. These five CSs are in the order of 1 to 5 (good to very poor), and represent the bridge condition ratings from $100 \%$ to $20 \%$ in a descending order (Figure 3 ).

While the condition index for BMS raw data consists of five CSs, the NBI raw data consists of nine different CSs (Figure 4). The acceptable numerical scale for the Artificial Neural Network (ANN) is ranged from -1 to 1 . Therefore a calibration procedure is required for the bridge condition datasets to fit into the 
BPM by using typical ANN input environment. This is presented in Figures 3 and 4 for BMS and NBI, respectively.

$\mathrm{Tu}$ [15]'s study has indicated that an empirical process is required to determine best possible combinations of the parameters such as learning rates $(l r)$ and momentum coefficient $(m c)$. The BPM-generated condition ratings for each year contain 66 spectra of rating numbers per CS [4], which is a combination of the learning rates (lr) (i.e. lr: 0.0 to 0.5 @ 0.1 increment, resulting in 6 lr), and momentum coefficients $(\mathrm{mc})$ (mc: 0.0 to $1.0 @ 0.1$ increment resulting in $11 \mathrm{mc}$ ) in the Neural Network configurations. Accordingly, the number of prediction in each year is also 66. Figure 5 shows the BPM results using the BMS raw data. For each year, there are 66 prediction results being derived from the combined number of $l r$ and $m c$ in the neural network. The symbols (66 points each year) represent the total number of element quantity (350 components) for that particular year. Between 1996 and 2004, the average quantity in CS1, CS2 and CS3 for Element \#234 is about $80 \%, 16.2 \%$ and $3.8 \%$ of the total element quantity, respectively. As seen in Figure 5, the historical condition ratings (from 1968 to 1994) generated by the BPM are in three different proportions of the condition state. The historical condition ratings shown in Figure 5(c) indicate that $3.8 \%$ of the total elements have historically fluctuated more than other CSs. This implies that MR\&R activities on these $3.8 \%$ of elements have been performed previously.

To validate the results of the BPM using the forward comparison technique, the generated backward prediction results (1968-1994) are used as input datasets to generate the condition ratings for the present years (1996-2004). It should be noted that the same BPM mechanism is applied for prediction in the forwarddirection (years 1996-2004). Two time periods (1968-1994 and 1996-2004) of the known non-bridge factors and one period (1968-1994) of the known generated condition ratings are used to predict the condition ratings for years (1996-2004). The forward prediction results (1996-2004) based on the BPM-generated condition ratings are then directly compared with the existing condition rating datasets (1996-2004). The prediction errors are calculated by averaging the differences between the forward prediction results and the actual BMS condition ratings. Table 2 shows the final results from the BPM and their prediction errors. The yearly average prediction errors are less than $\pm 10 \%$ which is considered 
acceptable. Therefore, the BPM generated historical condition ratings (19661994) can be used with confidence as historical BMS input data.

\subsection{NBI condition ratings as BPM input}

With a longer period of historical data provided by NBI, it is possible to measure the performance of the BPM prediction without any difficulties. Note that only a backward comparison is performed to verify the results generated by the BPM. Note also that only the condition ratings of the bridge deck are presented as BPM training inputs and outputs (from 1968 to 1994 in a 2-year increment) [16]. Additionally, assumed condition rating (i.e. excellent condition state) in 1966, when the bridge was built, is used [16]. As a result, the historical condition ratings are generated from 1968 to 1994 in a 2-year increment, as shown in Figure 6. Figure 7 illustrates the backward comparisons between the average BPM results and the existing NBI data. The prediction errors are calculated by averaging the differences between the BPM-generated condition ratings and the actual NBI data. As shown in the figure, the prediction errors in each year are less than $\pm 10 \%$ which is considered acceptable.

Evidently, by using the BPM-generated historical condition ratings, the prediction accuracies of the currently used typical deterioration modelling techniques can be improved. Notwithstanding this finding, it is still necessary to ascertain the efficiency of such generated historical data. To achieve this, the generated historical condition ratings are examined in relation to specific prediction techniques commonly used in the typical deterioration models [16].

\section{Typical bridge deterioration models}

Many research studies on bridge deterioration models have been carried out with an attempt to improve the reliability of BMS outcomes. Nonetheless, it has been emphasised that successful achievement of the analysis using these models remains highly dependent on the quality and sufficiency of data gathered [17]. According to Morcous et al [18], currently used typical bridge deterioration models can be categorised as deterministic, stochastic and artificial intelligence. In this paper, the first two modelling techniques are examined as they are popularly used in many current BMSs. Generally, a deterministic model predicts the deterioration of a bridge using a particular algorithm, while a stochastic model 
considers that the actual deterioration rate is unknown and there is a probability that the bridge will deteriorate at a specific rate [19].Amongst the deterministic models, regression analysis is a methodology widely used in many BMSs [17], whereas the Markov-based model is considered as one of the most common methods adopted by the stochastic techniques [20].

As described above, the BPM outcomes can lead to improved prediction accuracy. In the following section, evaluations of the prediction accuracies obtained from the linear and non-linear regression analyses, as well as the Markov-based model are presented. In general, the determination of a functional form of an equation that could fit particular datasets (also referred to as a performance curve) is considered as crucial part of a regression modelling [19]. As for linear regression, this function is expressed by a simple linear equation; whereas in non-linear regression, this function is characterised as a polynomial form of second or higher orders. In this study, following the work of Jiang and Sinha [3], a third-order polynomial model is used to determine long-term deterioration of bridge condition ratings.

$\mathrm{C}(\mathrm{t})=\beta_{0}+\beta_{1} \mathrm{t}_{\mathrm{i}}+\beta_{2} \mathrm{t}_{\mathrm{i}}^{2}+\beta_{3} \mathrm{t}_{\mathrm{i}}^{3}+\alpha_{\mathrm{i}}$

where $\mathrm{C}(\mathrm{t})=$ condition rating of a bridge at age $\mathrm{t} ; \mathrm{t}_{\mathrm{i}}=$ bridge age; $\alpha_{\mathrm{i}}=$ error term; and $\beta_{0}=$ recorded condition rating of a new bridge.

For the Markov-based model, generating a reliable transition probability is a key component. Percentage prediction and expected-value methods are two popular techniques which have been employed to generate transition probabilities in many state-of-art BMSs. Note that in estimating the transition probabilities, the percentage prediction method requires at least two consecutive condition records without any maintenance interventions, for a large number of bridge components at different condition states $[21,22]$. As a consequence, the available condition ratings used in this study, which do not satisfy the above condition, are unable to be used by the percentage prediction method. Therefore, the expected-value method is employed to estimate the transition probabilities of a bridge component. This is achieved by solving a non-linear optimisation objective function that minimises the difference between the overall condition ratings $A(t)$ and the expected condition ratings $E(t)$ resulted from the Markov-chain model. The overall condition ratings are generated using an Elman Neural Network (ENN) 
[23]. The non-linear objective function aimed at minimising the absolute difference between $A(t)$ and $E(t)$ may be expressed as follows:

$\operatorname{Min} \sum_{t=1}^{N}|A(t)-E(t)|$ subject to $0 \leq P(i) \leq 1, i=1,2,3 \ldots ., U$.

where $\mathrm{N}=$ the number of years in one age group; $\mathrm{U}=$ the number of unknown probabilities; $A(t)=$ the condition ratings at time $\mathrm{t}$, generated by the ENN; $E(t)=$ the condition ratings at time $\mathrm{t}$, estimated by the Markov-chain method.

\section{Comparison of deterioration model predictions}

\subsection{BPM-generated condition ratings using BMS raw data}

The long-term predictions of element \#234 due to both the regression analysis (deterministic) and the Markov-based model(stochastic) are carried out using different sets of BPM-generated condition ratings, i.e. 4 (from 1978 to 1984) being the minimum and 9 (from 1978 to 1994) being the maximum. Table 3 summarises the input data for prediction using the regression analysis and the Markov-based model. Also shown in the table are the numbers of predicted data used for comparison with the existing BMS condition ratings. Note that initially 14 missing historical condition ratings (from 1968 to 1994) are generated by the BPM. For the non-linear regression analysis, the polynomial degree of regression function must be less than or equal to the number of input minus 1 . In this study, a $3^{\text {rd }}$-order polynomial function is used, which is a typical process in the most deterioration models in BMS. Therefore the minimum number of dataset must be 4 (from year 1978 to 1984) which are taken from the abovementioned 9 condition ratings.

Figure 8 presents the prediction outcomes resulted from both linear and non-linear regression analyses based on 4 historical data records (from year 1978 to 1984) generated by the BPM. The average prediction errors of the linear regression are obtained by averaging the differences between the condition ratings of the existing BMS condition ratings and the prediction data from year 1996 to 2004. Similar method is employed to calculate the average prediction errors for the nonlinear regression; however, only 4 prediction data (from year 1996 to 2002) are available for comparison with the existing condition ratings. The average prediction errors of the linear and non-linear regressions are found to be $3.5 \%$ and 
$45.4 \%$, respectively. The large discrepancy resulted from the non-linear regression technique is due to the very limited number of input data. Such a limitation has a much higher impact on the non-linear regression than the linear method.

Figure 9 shows the prediction results based on 9 BPM-generated historical data records (from year 1978 to 1994). As described earlier, BPM based historical condition ratings are generated as 66 combinations of learning rates and momentum coefficients. In order for these results to be used in the regression analysis, the 66 combinations in each year from 1968 to 1994 are averaged to represent the individual condition rating records. Subsequently, the existing BMS condition ratings and the BPM-based prediction results are compared and the prediction accuracy is evaluated. Following the similar approach mentioned above, the average prediction errors between the generated condition ratings and the existing BMS condition ratings are calculated for both the linear and nonlinear regression models, which are $1.5 \%$ and $4.7 \%$, respectively. This demonstrates much improved predictions by the regression methods, when the number of BPM-generated input data is increased.

Figures 10 and 11 present the long-term prediction results of the Markov-based model based on 4 and 9 historical data records generated by the BPM.

Theoretically, the Markov-based model predicts bridge condition ratings using the probabilities of the bridge condition transition. These probabilities are characterised in a matrix type, namely, the transition probability matrix. Tables 4 and 5 present the transition probabilities which are produced by Equation 2 using 4 and 9 BPM-generated historical records. If the current state of bridge conditions or the initial state is known, the condition from one rating to another can be forecasted through multiplication of the original state vector and the transition probability matrix [3]. The average prediction errors of the Markov-based model are also obtained by averaging the differences between the condition ratings of the existing BMS condition ratings and the prediction data from year 1996 to 2004. These prediction errors are found to be $18.3 \%$ and $4.6 \%$, respectively using 4 and 9 BPM-generated input data.

Table 6 summarises the prediction errors using the linear regression, the nonlinear regression and the Markov-based techniques when incorporating the BPMgenerated condition ratings. It is evident that the prediction errors decrease as more input data become available. In the case of linear regression, the average 
error of $3.5 \%$ from the prediction using 4 generated condition ratings decreases to $1.5 \%$ when 9 generated condition ratings are used. Similarly for non-linear regression, the prediction error decreases from $45.4 \%$ to $4.7 \%$ when the number of input datasets increases. As for the Markov-based model, the prediction error decreases from $18.3 \%$ to $4.6 \%$.

\subsection{BPM-generate condition ratings using NBI data}

The long-term predictions of the bridge deck from both deterministic and stochastic models are also carried out using 4 selected NBI datasets $(1976,78,82$ and 84) and 9 out of 14 BPM-generated condition ratings (years 1968-1984) as input data, as given in Table 7. Also shown in the table are the numbers of predicted data used for comparison with the existing NBI data.

As presented in Figure 12, the average prediction errors of the linear regression using 4 NBI data are obtained by averaging the differences between the condition ratings of the existing NBI data and the prediction data from 1986 to 2004, with the exception of 1990 due to the NBI data being unavailable. Similar method is employed to calculate the average prediction errors of the non-linear regression, except that this calculation is based on only two valid prediction data, i.e. years 1986 and 1988. Results indicate that the average prediction errors of the linear and non-linear regressions are $33.3 \%$ and $25.6 \%$, respectively. It should be noted that the unrealistic pattern of deterioration as a result of the non-linear regression technique further confirms the limitation of the deterministic technique when only a very small number of condition rating data is available.

Figure 13 illustrates the prediction results based on 9 historical data generated by the BPM, as 66 combinations of learning rates and momentum coefficients. These combinations in each of the year 1968 to 1984 are averaged to represent the individual condition rating records to be used in the regression analysis.

Subsequently, the existing NBI records and the BPM-generated historical data are compared to evaluate the prediction accuracy. Following the similar approach mentioned above, the average prediction errors between the generated condition ratings and the NBI records are calculated for both the linear and non-linear regression models. This yields the average prediction errors of $6.6 \%$ and $9.0 \%$, respectively. 
For the Markov-based (stochastic) model, again 4 NBI data and 9 BPM-generated data are used as input for the bridge deck to predict the future condition ratings. The predicted results are compared with the existing NBI dataset to validate the prediction accuracy. Tables 8 and 9 present the generated transition probabilities based on 4 NBI data and 9 BPM-generated data. Figures 14 and 15 illustrate the long-term prediction results using the transition probabilities from Tables 8 and 9 . As indicated in the figures, the predicted condition ratings using 9 data input is more accurate than those using 4 data input. The average prediction errors between the predicted condition ratings and the existing NBI records from year 1986 to 2004 are found to be $24.5 \%$ for 4 NBI input data and $12.3 \%$ for 9 BPMgenerated input data.

Table 10 summarises the prediction errors using 4 NBI records and 9 BPMgenerated condition ratings, for the linear and non-linear regression techniques, as well as the Markov-based model. It is evident in the table that, for all these techniques, the prediction errors significantly decrease as more input data become available. In the case of linear regression, the average error of $33.3 \%$ from using 4 NBI records decreases to $6.6 \%$ when using 9 BPM-generated condition ratings. Similarly for the case of non-linear regression, the prediction error decreases from $25.6 \%$ to $9.0 \%$ when the number of input datasets increases. For the Markovbased model, the prediction error decreases from $24.5 \%$ to $12.3 \%$ when using 9 BPM-generated condition ratings. The above findings indicate that the amount of datasets is essential for numerical prediction methods to gain dependable prediction results. The results also suggest that, in both deterministic and stochastic models, the use of historical data generated by the BPM technique can improve the prediction accuracy. This reinforces the applicability of the BPM in generating missing historical condition ratings that are capable of providing a basis for more reliable predictions of future bridge conditions.

\section{Discussion and conclusion}

The performance of BMSs for optimal MR\&R strategy relies heavily on the bridge deterioration models, which in turn depends on the quality and sufficiency of the inspection data. The lack of historical bridge condition ratings is a major problem encountered by the current deterioration modelling techniques to achieve reliable predictions of future bridge conditions. To overcome this drawback, the 
Backward Prediction Model (BPM) has been developed as a means to assist in generating unavailable historical condition data, which was achieved by correlating the existing bridge condition dataset with the non-bridge factors including passenger vehicle, truck and total number of vehicles, highest temperature, local city population and state population growth. Using the nonbridge factors, 14 historical condition rating records (from 1968 to 1994) are generated. To ensure adequate quality of such generated data, future prediction results using the generated data are compared with five existing condition data (from 1996 to 2004 in a 2-year increment) and backward prediction results are directly compared with the available NBI condition ratings. For both the linear and non-linear regression (deterministic) modelling scenarios, the average prediction errors using 9 BPM-generated historical condition records are found to be less than those using 4 BPM-generated records (decreased by $26.7 \%$ in linear and $16.6 \%$ in non-linear regressions) and $4 \mathrm{NBI}$ records. Hence, using the BPM to generate more historical condition data helps to improve prediction accuracies. For the Markov-based (stochastic) model, the Elman Neural Network is employed to generate the performance curve of a typical bridge component based on 4 and 9 BPM-generated input data. The results indicate that using 9 BPM-generated records as input data for the Markov-based model can provide more accurate prediction results than using 4 BPM-generated records. The average prediction errors are decreased by $12.2 \%$ when the number of input data increases from 4 to 9 BPM-generated data.

Comparisons between the currently available and typical bridge deterioration models using different numbers of BPM-generated condition data demonstrate that the prediction errors decrease when the amount of input data increases. This provides further confirmation for the effectiveness of the BPM. To minimise the limitations with the existing deterministic and stochastic models as identified in this study and to provide useful solutions for the asset management practitioners and researchers, further study is essential and should aim at developing a robust long-term bridge deterioration model that fully utilises the benefits of the BPM in practical applications.

Acknowledgment The resources used for the present study were provided by the Maryland State Department of Transportation, U.S. The authors wish to thank Messrs Earle Freedman and Matt Zulkowski for providing information requested. 


\section{References}

1. Aktan AE, Catbas FN, Grimmelsman KA, Tsikos CJ (2000) Issue in infrastructure health monitoring for management. J Eng Mech 126:711-724.

2. Madanat S, Ibrahim WHW (1995a) Poisson regression model of infrastructure transition probabilities. J Tran Eng 121:267-272.

3. Jiang Y, Sinha KC (1989) Bridge service life prediction model using the Markov Chain. J Tran Res Board 24-30.

4. Lee JH, Sanmugarasa K, Blumenstein M, Loo YC (2008) Improving the reliability of a Bridge Management System (BMS) using an ANN-based Backward Prediction Model (BPM). J Auto Construct 17(6):758-772.

5. Godar B, Vassie R (1999) Review of existing BMS and definition of inputs for the proposed BMS. Deliverable D4 BRIME Report. PL97-2220.

6. Mishalani RG, McCord MR (2006) Infrastructure condition assessment, deterioration modeling, and maintenance decision making: methodological advances and practical considerations. J Infrastruct Syst 12(3):145-146.

7. Mauch M, Madanat S (2001) Semiparametric hazard rate models of reinforced concrete bridge deck deterioration. J Infrastruct Syst 7(2):49-57.

8. Madanat S, Mishalani R, Ibrahim WHW (1995b) Estimation of infrastructure transition probabilities from condition rating data. J Infrastruct Syst 1(2):120-125.

9. Agrawal AK, Qian G, Kawaguchi A, Lagace S, Delisle R, Kelly B, Weykamp P, Conway T, Dublin E (2006) Deterioration rates of typical bridge elements in New York. ASCE.

10. DeStefano PD, Grivas DA (1998) Method for estimating transition probability in bridge deterioration models. J Infrastruct Syst 4(2):56-62.

11. Madanat SM, Karlaftis MG, McCarthy PS (1997) Probabilistic infrastructure deterioration models with panel data. J Infrastruct Syst 3(1):120-125.

12. Morcous G, Rivard H, Hanna AM (2000) Case-based reasoning system for bridge management. Comp Civil and Building Eng. 1363-1370.

13. Son JB, Lee JH, Blumenstein M, Loo YC, Guan H and Panuwatwanich K (2009a) Improving reliability of Bridge deterioration model using generated missing condition ratings. 3rd Inter Conf on Constr Eng and Management (ICCEM), Jeju, S. Korea.

14. Queensland department of main road (2004) Bridge inspection manual. Registration number 80.640, Queensland, Department of Main Roads, Transport Technology Division.

15. Tu VT (1996) Advantage and disadvantage of using Artificial Neural Networks versus logistic regression for predicting medical outcomes. J Clinic epidemiology 49(11):1225-1231.

16. Son JB, Lee JH, Blumenstein M, Loo YC, Guan H and Panuwatwanich K (2009b) Generating Historical Condition Ratings for the Reliable Prediction of Bridge Deteriorations. 33rd Inter Association for Bridge and Structural Eng (IABSE) Symposium, Thailand.

17. Kleywegt AJ, Sinha KC (1994) Tools for bridge management data analysis. Trans Res Circ 423:16-26. 
18. Morcous G, Rivard H, Hanna AM (2002) Modelling bridge deterioration using case-based reasoning. J Infrastruct Syst 8(3):86-95.

19. Austroads (2002) Bridge management systems - the state of the art. Austroads: AP-R198/02, 23.

20. Micevski T, Kuczera G, Coombes P (2002) Markov model for storm water pipe deterioration. J Infrastruct Syst 8(2):49-56.

21. Morcous G (2006) Performance Prediction of Bridge Deck Systems Using Markov Chains. J Perform Constr Facil 20(2):146-155.

22. Bu GP, Lee JH, Guan H, Blumenstein M, Loo YC (2012a) Development of an integrated method for probabilistic bridge deterioration modelling. J Perform Constr Facil (Accepted for publication 14/11/2012).

23. Bu GP, Lee JH, Guan H, Loo YC, Blumenstein M (2012b) Long-term performance of bridge elements using integrated deterioration method incorporating Elman Neural Network. Applied Mechanics and Materials 204-208:1980-1987. 


\section{List of figures}

Fig 1 Mechanism of BPM [4]

Fig 2 NBI raw data (Deck, Bridge \#0301xxxx1)

Fig 3 Condition index for BMS condition ratings

Fig 4 Condition index for NBI condition ratings

Fig 5 BPM results using BMS raw data (Element \#234, Bridge \#0301xxxx1)

Fig 6 BPM results using NBI raw data (Bridge \#0301xxxx1)

Fig 7 Comparison between BPM outcomes and actual NBI data (Bridge \#0301xxxx1)

Fig 8 Prediction results using 4 sets of BPM-generated records based on BMS raw data in deterministic model

Fig 9 Prediction results using 9 sets of BPM-generated records based on BMS raw data in deterministic model

Fig 10 Prediction results using 4 sets of BPM-generated records based on BMS raw data in Markov-based (stochastic) model

Fig 11 Prediction results using 9 sets of BPM-generated records based on BMS raw data in Markov-based (stochastic) model

Fig 12 Prediction results using 4 NBI raw data

Fig 13 Prediction results using 9 sets of BPM-generated records based on NBI raw data

Fig 14 Prediction results using 4 sets of NBI raw data in Markov-based (stochastic) model

Fig 15 Prediction results using 9 sets of BPM-generated records based on NBI raw data in Markov-based (stochastic) model 
Table 1 BMS raw data of actual condition ratings (Element \#234 on Bridge \#0301xxxx1)

\begin{tabular}{ccccccc}
\hline $\begin{array}{c}\text { Year of } \\
\text { inspection }\end{array}$ & $\begin{array}{c}\text { Total Qty. } \\
(\%)\end{array}$ & $\begin{array}{c}\text { CS1 Qty. } \\
(\%)\end{array}$ & $\begin{array}{c}\text { CS2 Qty. } \\
(\%)\end{array}$ & $\begin{array}{c}\text { CS3 Qty. } \\
(\%)\end{array}$ & $\begin{array}{c}\text { CS4 Qty. } \\
(\%)\end{array}$ & $\begin{array}{c}\text { CS5 Qty. } \\
(\%)\end{array}$ \\
\hline 1996 & $350(100)$ & $280(80)$ & $50(14)$ & $20(6)$ & 0 & 0 \\
1998 & $350(100)$ & $280(80)$ & $50(14)$ & $20(6)$ & 0 & 0 \\
2000 & $350(100)$ & $280(80)$ & $50(14)$ & $20(6)$ & 0 & 0 \\
2002 & $350(100)$ & $283(80)$ & $67(19)$ & $3(1)$ & 0 & 0 \\
2004 & $350(100)$ & $283(80)$ & $67(19)$ & $3(1)$ & 0 & 0 \\
\hline
\end{tabular}


Table 2 Prediction errors of the proposed BPM using forward comparison (Bridge \#0301xxxx1)

\begin{tabular}{|c|c|c|c|c|c|c|c|c|}
\hline & & $\begin{array}{c}\text { Total } \\
(\%)\end{array}$ & $\begin{array}{l}\text { CS1 } \\
(\%)\end{array}$ & $\begin{array}{l}\text { CS2 } \\
(\%)\end{array}$ & $\begin{array}{l}\text { CS3 } \\
(\%)\end{array}$ & $\begin{array}{l}\text { CS4 } \\
(\%)\end{array}$ & $\begin{array}{l}\text { CS5 } \\
(\%)\end{array}$ & $D$ \\
\hline \multirow{3}{*}{1996} & A & 100.0 & 86.0 & 12.0 & 2.0 & 0.0 & 0.0 & \multirow{3}{*}{$2.4 \%$} \\
\hline & B & 100.0 & 80.0 & 14.3 & 5.7 & 0.0 & 0.0 & \\
\hline & $\mathrm{C}$ & & 6.0 & 2.3 & 3.7 & 0.0 & 0.0 & \\
\hline \multirow{3}{*}{1998} & $\mathrm{~A}$ & 100.0 & 86.0 & 12.0 & 2.0 & 0.0 & 0.0 & \multirow{3}{*}{$2.4 \%$} \\
\hline & B & 100.0 & 80.0 & 14.3 & 5.7 & 0.0 & 0.0 & \\
\hline & $\mathrm{C}$ & & 6.0 & 2.3 & 3.7 & 0.0 & 0.0 & \\
\hline \multirow{3}{*}{2000} & $\mathrm{~A}$ & 100.0 & 86.0 & 12.0 & 2.0 & 0.0 & 0.0 & \multirow{3}{*}{$2.4 \%$} \\
\hline & B & 100.0 & 80.0 & 14.3 & 5.7 & 0.0 & 0.0 & \\
\hline & $\mathrm{C}$ & & 6.0 & 2.3 & 3.7 & 0.0 & 0.0 & \\
\hline \multirow{3}{*}{2002} & A & 100.0 & 86.0 & 12.0 & 2.0 & 0.0 & 0.0 & \multirow{3}{*}{$2.84 \%$} \\
\hline & B & 100.0 & 80.0 & 19.1 & 0.9 & 0.0 & 0.0 & \\
\hline & $\mathrm{C}$ & & 6.0 & 7.1 & 1.1 & 0.0 & 0.0 & \\
\hline \multirow{3}{*}{2004} & A & 100.0 & 86.0 & 12.0 & 2.0 & 0.0 & 0.0 & \multirow{3}{*}{$2.84 \%$} \\
\hline & B & 100.0 & 80.0 & 19.1 & 0.9 & 0.0 & 0.0 & \\
\hline & $\mathrm{C}$ & & 6.0 & 7.1 & 1.1 & 0.0 & 0.0 & \\
\hline
\end{tabular}

A-results of forward prediction; B-actual condition ratings;

C-difference between A and B; and D-average of difference 
Table 3 Input data and predicted data for comparison with existing BMS condition rating (Bridge \#0301xxxx1)

\begin{tabular}{|c|c|c|}
\hline Prediction techniques & $\begin{array}{l}\text { Input data for } \\
\text { prediction }\end{array}$ & $\begin{array}{c}\text { Predicted data compared with existing condition } \\
\text { ratings }\end{array}$ \\
\hline \multirow{2}{*}{ Linear regression } & $\begin{array}{l}4 \text { BPM data } \\
(1978-1984)\end{array}$ & 5 data (1996-2004) \\
\hline & $\begin{array}{l}9 \text { BPM data } \\
(1978-1994)\end{array}$ & 5 data (1996-2004) \\
\hline \multirow{2}{*}{ Non-linear regression } & $\begin{array}{l}4 \text { BPM data } \\
(1978-1984)\end{array}$ & 4 data (1996-2002) \\
\hline & $\begin{array}{l}9 \text { BPM data } \\
(1978-1994)\end{array}$ & 5 data (1996-2004) \\
\hline \multirow{2}{*}{ Markov-based model } & $\begin{array}{l}4 \text { BPM data } \\
(1978-1984)\end{array}$ & 5 data (1996-2004) \\
\hline & $\begin{array}{l}9 \text { BPM data } \\
(1978-1994)\end{array}$ & 5 data (1996-2004) \\
\hline
\end{tabular}


Table 4 Transition probabilities (generated 4 BPM data as input using BMS data)

\begin{tabular}{lccccc}
\hline Age group & 1 & 2 & 3 & 4 & 5 \\
\hline$(12-17)$ & 0.930 & 1.000 & 1.000 & 1.000 & 1.000 \\
$(18-22)$ & 0.945 & 1.000 & 1.000 & 1.000 & 1.000 \\
$(23-27)$ & 0.963 & 0.882 & 1.000 & 1.000 & 1.000 \\
$(28-32)$ & 0.955 & 0.898 & 1.000 & 1.000 & 1.000 \\
$(33-37)$ & 0.948 & 0.941 & 0.967 & 1.000 & 1.000 \\
$(38-42)$ & 0.909 & 0.969 & 1.000 & 1.000 & 1.000 \\
$(43-47)$ & 0.909 & 0.969 & 1.000 & 1.000 & 1.000 \\
$(48-52)$ & 0.909 & 0.969 & 1.000 & 1.000 & 1.000 \\
\hline
\end{tabular}


Table 5 Transition probabilities (generated 9 BPM data as input using BMS data)

\begin{tabular}{lccccc}
\hline Age group & 1 & 2 & 3 & 4 & 5 \\
\hline$(12-17)$ & 0.960 & 1.000 & 1.000 & 1.000 & 1.000 \\
$(18-22)$ & 0.973 & 1.000 & 1.000 & 1.000 & 1.000 \\
$(23-27)$ & 0.978 & 1.000 & 1.000 & 1.000 & 1.000 \\
$(28-32)$ & 0.979 & 1.000 & 1.000 & 1.000 & 1.000 \\
$(33-37)$ & 0.979 & 1.000 & 1.000 & 1.000 & 1.000 \\
$(38-42)$ & 0.977 & 1.000 & 1.000 & 1.000 & 1.000 \\
$(43-47)$ & 0.974 & 1.000 & 1.000 & 1.000 & 1.000 \\
$(48-52)$ & 0.986 & 0.948 & 1.000 & 1.000 & 1.000 \\
\hline
\end{tabular}


Table 6 Summary of prediction errors using different sets of BPM-generated data

\begin{tabular}{cccccccc}
\hline \multirow{2}{*}{$\begin{array}{c}\text { Prediction } \\
\text { techniques }\end{array}$} & $\begin{array}{c}\text { Number } \\
\text { of input }\end{array}$ & \multicolumn{3}{c}{ Difference between prediction and existing BMS condition } & $\begin{array}{c}\text { Prediction } \\
\text { ratings }\end{array}$ \\
\cline { 3 - 7 } & data & 1996 & 1998 & 2000 & 2002 & 2004 & (Mean) \\
\hline Linear & 4 & $2.5 \%$ & $2.8 \%$ & $3.1 \%$ & $4.3 \%$ & $4.6 \%$ & $3.5 \%$ \\
regression & 9 & $0.8 \%$ & $1.0 \%$ & $1.1 \%$ & $2.3 \%$ & $2.4 \%$ & $1.5 \%$ \\
\hline Non-linear & 4 & $24.7 \%$ & $36.4 \%$ & $50.9 \%$ & $69.5 \%$ & - & $45.4 \%$ \\
regression & 9 & $2.7 \%$ & $3.4 \%$ & $4.2 \%$ & $6.1 \%$ & $7.0 \%$ & $4.7 \%$ \\
\hline Markov- & 4 & $14.0 \%$ & $15.8 \%$ & $17.6 \%$ & $21.2 \%$ & $22.7 \%$ & $18.3 \%$ \\
based model & 9 & $2.9 \%$ & $3.4 \%$ & $3.9 \%$ & $6.1 \%$ & $6.5 \%$ & $4.6 \%$ \\
\hline
\end{tabular}


Table 7 Input data and predicted data for comparison with existing NBI data (Bridge \#0301xxxx1)

\begin{tabular}{|c|c|c|}
\hline $\begin{array}{l}\text { Prediction } \\
\text { techniques }\end{array}$ & Input data for prediction & $\begin{array}{l}\text { Predicted data compared with existing } \\
\qquad \text { NBI data }\end{array}$ \\
\hline \multirow{3}{*}{$\begin{array}{l}\text { Linear } \\
\text { regression }\end{array}$} & 4 NBI data $(1976,1978,1982$ and & 9 data $(1986,1988$ and from $1992-$ \\
\hline & 1984) & 2004) \\
\hline & 9 BPM data (from 1968-1984) & $\begin{array}{c}9 \text { data }(1986,1988 \text { and from } 1992- \\
2004)\end{array}$ \\
\hline \multirow{2}{*}{$\begin{array}{l}\text { Non-linear } \\
\text { regression }\end{array}$} & $\begin{array}{c}4 \text { NBI data }(1976,1978,1982 \text { and } \\
1984)\end{array}$ & 2 data (1986 and 1988) \\
\hline & 9 BPM data (from 1968-1984) & 2 data (1986 and 1988) \\
\hline \multirow{2}{*}{$\begin{array}{l}\text { Markov- } \\
\text { based model }\end{array}$} & $\begin{array}{c}4 \text { NBI data }(1976,1978,1982 \text { and } \\
1984)\end{array}$ & $\begin{array}{l}9 \text { data }(1986,1988 \text { and from } 1992- \\
2004)\end{array}$ \\
\hline & 9 BPM data (from 1968-1984) & $\begin{array}{c}9 \text { data }(1986,1988 \text { and from } 1992- \\
2004)\end{array}$ \\
\hline
\end{tabular}


Table 8 Transition probabilities (input using 4 NBI data)

\begin{tabular}{lccccccccc}
\hline Age group & 1 & 2 & 3 & 4 & 5 & 6 & 7 & 8 & 9 \\
\hline $10-15$ & 0.000 & 0.789 & 1.000 & 1.000 & 1.000 & 1.000 & 1.000 & 1.000 & 1.000 \\
$16-20$ & 0.000 & 0.887 & 0.738 & 1.000 & 1.000 & 1.000 & 1.000 & 1.000 & 1.000 \\
$21-25$ & 0.000 & 0.896 & 0.757 & 0.794 & 0.787 & 0.994 & 1.000 & 1.000 & 1.000 \\
$26-30$ & 0.000 & 0.900 & 0.819 & 0.764 & 0.639 & 0.537 & 0.148 & 0.994 & 1.000 \\
$31-35$ & 0.000 & 0.880 & 0.850 & 0.800 & 0.698 & 0.492 & 0.000 & 0.724 & 1.000 \\
$36-40$ & 0.000 & 0.863 & 0.845 & 0.766 & 0.655 & 0.574 & 0.001 & 0.975 & 1.000 \\
$41-45$ & 0.000 & 0.841 & 0.852 & 0.736 & 0.641 & 0.573 & 0.002 & 1.000 & 1.000 \\
\hline
\end{tabular}


Table 9 Transition probabilities (generated 9 BPM data as input using NBI data)

\begin{tabular}{lccccccccc}
\hline Age group & 1 & 2 & 3 & 4 & 5 & 6 & 7 & 8 & 9 \\
\hline $2-7$ & 0.129 & 0.991 & 0.890 & 1.000 & 1.000 & 1.000 & 1.000 & 1.000 & 1.000 \\
$8-12$ & 0.602 & 0.958 & 0.785 & 0.817 & 0.984 & 1.000 & 1.000 & 1.000 & 1.000 \\
$13-17$ & 0.722 & 0.952 & 0.770 & 0.732 & 0.781 & 0.975 & 1.000 & 1.000 & 1.000 \\
$18-22$ & 0.806 & 0.944 & 0.807 & 0.708 & 0.534 & 0.785 & 0.999 & 1.000 & 1.000 \\
$23-27$ & 0.842 & 0.940 & 0.816 & 0.710 & 0.513 & 0.662 & 0.832 & 1.000 & 1.000 \\
$28-32$ & 0.862 & 0.934 & 0.821 & 0.720 & 0.536 & 0.647 & 0.755 & 1.000 & 1.000 \\
$33-37$ & 0.847 & 0.941 & 0.809 & 0.671 & 0.489 & 0.628 & 0.808 & 1.000 & 1.000 \\
$38-42$ & 0.836 & 0.945 & 0.802 & 0.669 & 0.488 & 0.627 & 0.808 & 1.000 & 1.000 \\
\hline
\end{tabular}


Table 10 Summary of prediction errors using 4 NBI and 9 BPM-generated data

\begin{tabular}{ccccccccccccc}
\hline $\begin{array}{c}\text { Prediction } \\
\text { techniques }\end{array}$ & $\begin{array}{c}\text { Number } \\
\text { of input } \\
\text { data }\end{array}$ & \multicolumn{1}{c}{ Difference between prediction and existing BMS condition ratings } & $\begin{array}{c}\text { Prediction } \\
\text { error (Mean) }\end{array}$ \\
\hline & & & 1986 & 1988 & 1992 & 1994 & 1996 & 1998 & 2000 & 2002 & 2004 & \\
\hline Linear & 4 & $\%$ & 15.0 & 20.0 & 30.0 & 35.0 & 30.0 & 35.0 & 40.0 & 45.0 & 50.0 & 33.3 \\
regression & 9 & $\%$ & 3.4 & 5.2 & 8.9 & 10.7 & 2.6 & 4.4 & 6.3 & 8.1 & 10.0 & 6.6 \\
Non-linear & 4 & $\%$ & 10.4 & 40.7 & & & & & & & & 25.6 \\
regression & 9 & $\%$ & 6.8 & 11.2 & & & & & & & & \\
Markov- & 4 & $\%$ & 4.6 & 9.0 & 18.2 & 25.5 & 22.0 & 29.2 & 34.5 & 37.9 & 39.9 & 24.5 \\
based model & 9 & $\%$ & 2.8 & 5.7 & 12.5 & 15.9 & 9.1 & 12.1 & 15.0 & 17.6 & 20.1 & 12.3 \\
\hline
\end{tabular}

